\title{
Investigation, Examination, Control, and Anxiety
}

\section{Brian Bergen-Aurand}

This is a special issue on surveilled bodies, with five articles guest edited by Ira Allen, Assistant Professor of Rhetoric, Writing, and Digital Media Studies at Northern Arizona University and Assistant Editor of Screen Bodies. The question here is one of how screens and bodies are brought together through surveillance (visual and otherwise), how surveillance hails the body to attend to it (beckons us to catch a glimpse of here or there) even as it hides itself from the body, working to be noticed yet remaining unnoticed, in order to keep us "on our toes." In this light, surveillance is not only about investigating, examining, logging, and controlling the body but also about bringing the body into being as a body-to-be-surveilled, about interpolating the body into becoming evermore surveillable in ever-more granular ways.

On 27 October 2019, first-term US Representative Katie Hill (Democrat from California) resigned her seat in Congress in response to allegations of having inappropriate sexual relations with members of her staff. The case drew special attention from the media and critics because it also involved what has been called a "messy divorce," reports of polyamorous relationships, and "weaponized revenge porn" in the form of nude photographs released to humiliate the Congresswoman. As well, many have questioned why Hill was investigated under a new Congressional law designed to protect staff from unwanted sexual harassment after so many other revelations have arisen because of the \#metoo movement. The fact that it was a queer, female politician who was the first to fall, has not gone unnoticed by those watching the law. On 1 November 2019, Google announced that it was buying the wearables company Fitbit for $\$ 7.35$ a share or $\$ 2.1$ billion total. The offer has to be approved by invested parties on all sides. Immediately, stakeholders concerned over issues of privacy, corporeal user information, and data security raised their voices to object to the acquisition. That same day, Marketplace ran a story entitled "Behind the Curtain of the 'Ethical Algorithm'” that featured a report on a Science article scrutinizing an algorithm from United Health Group that hospitals use to decide which patients 
receive supplemental care and support. Researchers discovered that the software is racially biased despite not having race written into the code because race and cost are related when it comes to healthcare: white patients tend to seek more costly medical intervention. Thus, basing the algorithm on cost prejudiced the software against black patients. Critics have raised objections to the use of such "proxies" when encoding programs and have charged that the collection and deployment of big medical data can only remain ethical when it is filtered through socially responsible lenses rather than viewed solely with regard to the objective of "predictive accuracy."

In his introduction to the topic of ubiquitously surveilled bodies, Allen emphasizes that "no one is talking about what it means to be surveilled everywhere, from everywhere, in every way," and the three cases above reinforce the point he makes regarding the fact that surveillance is now the culture we inhabit. (To borrow a social justice trope: it has become the water we swim in.) Indeed, precisely because they do not all seem like instances of surveillance, these three incidences may well mark moments where we might recognize the multivariance of contemporary surveillance culture and the attendant anxiety that accompanies our having become ubiquitously surveilled bodies - if we are able to recognize it at all, as it has become both the foreground and the background of our present age. Precisely the fact that they might not all seem like surveillance in the first place, though, also marks these instances of data collection, manipulation, and dissemination as sites where we might open surveillance to scrutiny. If we do not necessarily recognize it when we see it, then we might learn something new about surveillance and respond to it differently when we do. Perhaps, such moments of recognition are exactly when we should say something because we have seen something.

Surveilled bodies come in all shapes and sizes, as do the devices through which we encounter them. In this issue, Allen and the other authors-Kellie Renee Marin, Laura A. Sparks, JWells, and Sarah Young-begin with the question of whether or not it is possible for our bodies to no longer be surveilled. Together, they ask whether there is any way out of corporeal surveillance, or if it has become (or if it has always already been) ubiquitous. In their individual articles, they question sites of security and witnessing, the temporality of torture, imprisonment, and digital identity formations. And, in the end, Damien Pfister responds to their analyses in a way that calls for additional questions and considerations - bringing us from the ubiquity of surveillance to a thinking of it granularity. Throughout these pieces, these authors return to the idea that "nowhere is not the panopticon" (or whatever dispositif succeeds it) and everywhere is the "exemplary project of the public-private governance partnership" that has become the age in which we live.

In addition to this significant consideration of surveilled bodies, issue 4.2 also contains an article on the filmmaking of Roger Jacoby and the queer- 
ness of alternative film processing by Benjamin Ogrodnik. As well, it includes Bilge Gölge's report on \#yogabody on Instagram and the complex relationship between social network services and responses to alternative body images across cultures. Finally, this issue closes with two reviews: John Stadler's assessment of Making Sex Public and Other Cinematic Fantasies by Damon R. Young and Mauro Carbone's Philosophy-Screens: From Cinema to the Digital Revolution. If, as Carbone surmises, the ubiquitous state of the present age is a constant low-hum anxiety maintained by our relationships with our screens, then a review of this book is a fitting close to this issue of Screen Bodies.

Brian Bergen-Aurand teaches in the Division of Arts \& Humanities and Digital Learning at Bellevue College, Washington, where he specializes in screen studies, ethics, embodiment, and humor studies. He is the founder and editor of the Screen Bodies journal, the editor of Comedy Begins with Our Simplest Gestures: Levinas, Ethics, and Humor (2017) and Transnational Chinese Cinema, Corporeality, Desire, and the Ethics of Failure (2014, with Mary Mazzilli and Hee Wai-Siam), and the author of Cinematic Provocations: Ethics, Justice, Embodiment, and Global Film (forthcoming 2020). Currently, he is developing (with Andrew Grossman) a study of global queer cinema.

Email: screenbodies@berghahnjournals.com 